\title{
Editor's Message to Special Issue on Applications and the Internet in Conjunction with Main Topics of SAINT 2012
}

\author{
HIROYUKI OHSAKI ${ }^{1, a)}$
}

It is our great pleasure to have the special issue of Journal of Information Processing (JIP) on "Applications and the Internet in Conjunction with Main Topics of SAINT 2012." The IEEE/IPSJ International Symposium on Applications and the Internet (SAINT) was intended to provide a forum for researchers in the Internet and its applications to discuss the current status, technical challenges, standards, fundamental issues, future services, and applications. This symposium covered a broad range of networking and computing technologies and advanced applications and services. SAINT 2012 was held in Izmir, Turkey on July 16-20, 2012. From 2013, SAINT will be merged into The Annual International Computer Software \& Applications Conference (COMPSAC), sponsored by IEEE Computer Society and also technically sponsored by IPSJ.

We received many submissions related to the Internet and its applications. Among 24 submissions, half of them were from authors of the papers presented at SAINT conferences and their workshops. Out of 24 submissions, 13 papers were accepted (i.e., $54 \%$ acceptance rate). In addition, we had an invited paper from Prof. Carl K. Chang of Iowa State University, USA, who has been the chair of the standing committee of COMPSAC. Consequently, we had 14 papers published in this special issue.

Finally, I greatly appreciate efforts and contributions made by all editors of this special issue.

\section{The Editorial Committee}

- Editor in-Chief:

Hiroyuki Ohsaki (Kwansei Gakuin University)

\section{- Editorial Board:}

Nariyoshi Yamai (Okayama University)

- Editorial Committee:

Dan Lin (Missouri University of Science and Technology)

Hen-I Yang (Iowa State University)

Susumu Ishihara (Shizuoka University)

Takashi Imaizumi (Chiba University)

Yasuo Okabe (Kyoto University)

1 Department of Informatics School of Science and Technology, Kwansei Gakuin University, Sanda, Hyogo 669-1337, Japan

a) ohsaki@kwansei.ac.jp http://www.lsnl.jp/
Eiji Kawai (NICT)

Atsushi Tagami (KDDI R\&D Labs)

Yuuichi Teranishi (NICT)

Motonori Nakamura (NII)

Yutaka Nakamura (Kyushu Institute of Technology)

Kazutoshi Fujikawa (Nara Institute of Science and Technol-

ogy)

Katsuyuki Yamazaki (Nagaoka University of Technology)

Takashi Yamanoue (Kagoshima University) 